\title{
Electroosmotic Flow Measurements in a Freely Suspended Liquid Film: Experiments and Numerical
}

\section{Simulations}

Abdulkadir Hussein Sheik, H.C. Hemaka Bandulasena*, Victor Starov, Anna Trybala

Department of Chemical Engineering, Loughborough University, Loughborough, LE11 3TU, UK.

*Corresponding author.

Email:H.C.H.Bandulasena@lboro.ac.uk, Tel.: +44 (0) 1509222515

Keywords: Electroosmotic flow, Free liquid film, Plateau border, Electrophoresis,

Abbreviations

$\mathrm{CMC}$ - Critical micelle concentration

CLSM - Confocal laser scanning microscopy

FEM - Finite Element Method

$\mu$-PIV - Micron-Resolution Particle Image Velocimetry

MTAB - Myristyltrimethylammonium bromide

SDS - Sodium dodecyl sulphate

Total

Table: 0

Figures: 5

Words: 4472

Abstract:

Fluid flow profiles in free liquid films stabilised by anionic and cationic surfactants under an external electric field were investigated. Depthwise velocity fields were measured at the mid region of the

Received: MONTH DD, YYY; Revised: MONTH DD, YYY; Accepted: MONTH DD, YYY

This article has been accepted for publication and undergone full peer review but has not been through the copyediting, typesetting, pagination and proofreading process, which may lead to differences between this version and the Version of Record. Please cite this article as doi: 10.1002/elps.201600549.

This article is protected by copyright. All rights reserved. 
free liquid film by confocal $\mu$-PIV and corresponding numerical simulations were performed using Finite Element Method (FEM) to model the system. Depthwise change in velocity profiles was observed with electroosmotic flow dominating in the vicinity of the gas-liquid and solid-liquid interfaces while backpressure drives fluid in the opposite direction at the core of the film. It was also found that the direction of the flow at various sections of the films depends on the type of surfactant used, but flow features remained the same. Numerical simulations predicted the flow profiles with reasonable accuracy; however, asymmetry of the actual film geometry caused deviations at the top half of the computational domain. Overall, electroosmotic flow profiles within a free liquid film is similar to that of the closed-end solid microchannel. However, the flow direction and features of the velocity profiles can be changed by selecting various types of surfactants. The free liquid films thickness was selected to match dimensions of foam Plateau border. Hence these findings will be useful in developing a separation system based on foam electrokinetics. 


\section{Introduction}

The ability to manipulate fluids in free liquid films by an external electric field is interesting academically and has several potential applications in novel separation approaches of biological molecules such as proteins and DNA. A free liquid film can be formed by suspending a small volume of liquid with a solid frame and stabilising the interfaces with surfactants. Foam mainly consists of liquid lamella, Plateau borders and vertices all of which can be considered as free liquid films. Foam is a dispersed system that consists of gas bubbles partitioned by liquid layers. Foams have a wide range of applications stretching from cosmetics to beverages and beyond [1]. A free liquid film can be used to model transport phenomena in foams.

Several investigations reported manipulation of fluid in free liquid films by various mechanisms. A liquid film motor has been reported by [2, 3], where a free liquid film placed in between two capacitively coupled plates is connected to a DC power source. The electric field applied to the liquid exerted a force on the induced free charges accumulated on the surface of the film and caused the fluid to undergo complex motion. In a similar study, electrodes were passed through the freely suspended liquid film, and an AC voltage was supplied to the terminals [4]. The vortices were induced within the film by electrohydrodynamic induction, and the fluid velocity depended on the frequency of the AC voltage and the thickness of the liquid film [4]. In [5], reverse drainage of a cylindrical soap bubble placed between two platinum plate electrodes was investigated. Foam stability is affected by liquid drainage due to gravity; hence reversing this effect could be beneficial in increasing the half-life of foam. In this study, electroosmotic flow pumped fluid from the bottom reservoir to the top of the film and the flow rate was found to be nonlinear with the applied electric field. Most importantly, the liquid film was not bound by solid surfaces; hence accommodated additional fluid within the column by film thickening $[5,6]$. Recently, another study investigated electrokinetic stabilisation of gravitational drainage in free liquid films [7]. They found that both 
anionic and cationic surfactants can stabilise foams irrespective to the direction of the electric field applied as electroosmotic flow induces pressure flow in the opposite direction.

In microfluidics devices, fluid flow can be controlled by external electric fields [2], and the resultant electrokinetic flow has its origin near the solid-liquid surfaces. Even though significant advances have been made in understanding electrokinetic transport at solid-liquid interfaces [8], electrokinetic phenomena at gas-liquid interfaces require further attention [5]. Electrokinetic flow in the case of gas-liquid interfaces differs from that of the solid-liquid interface because hydrodynamic boundary condition in the former is much more complicated [9]. For a gas-liquid interface, friction between ions and surfactant micelles in the liquid should be determined [6], and thermal capillary waves due to fluctuations of the free interfaces should be considered [10].

In this paper, we present flow field inside a free liquid film under an applied electric field experimentally and compare the results with numerical simulations. The effect of surfactant type, i.e. cationic and anionic, on the flow field is also investigated and compared with the numerical predictions. The dimensions of the liquid film are carefully selected to match a foam Plateau border, hence the findings of this study will be beneficial for understanding foam electrokinetics. To the best of our knowledge, detailed electroosmotic velocity profiles in free liquid films stabilised by various surfactants have not been reported in the literature.

\section{Material and Methods}

\subsection{Solution preparation and characterization}

All test solutions were prepared by dissolving either $53.6 \mathrm{mg}$ of cationic surfactant Myristyltrimethylammonium bromide (MTAB) or $70.9 \mathrm{mg}$ of anionic surfactant Sodium dodecyl sulphate (SDS) (both from Sigma-Aldrich, UK) in $30 \mathrm{~g}$ of $50 \% \mathrm{w} / \mathrm{w}$ glycerol made of Milli-Q water (15 
$\mathrm{M} \Omega . c m$ deionised water). The concentrations of the surfactants were just above critical micelle concentration (CMC). Glycerol was added to the solution to increase the stability of the liquid films and extend its lifetime by reducing the rate of drainage. A concentrated phosphate buffer solution was added to this mixture until the solution molarity reached $2 \mathrm{mM}$ to avoid generation of $\mathrm{pH}$ gradients. To aid with flow visualisation, $2 \mu \mathrm{m}$ fluorescent carboxylate-modified polystyrene particles (Sigma-Aldrich Ltd, UK) were added to the test solution to achieve a particle concentration of $0.003 \%$. Finally, an electrically neutral dye (Rhodamine B) was added to the test solution to determine the flow geometry by confocal laser scanning microscopy CLSM. The test solution had a $\mathrm{pH}$ of 7.25, and electric resistivity of $12.5 \mathrm{M} \Omega$. cm was measured using Thermo Scientific Orion Star A215. The density was measured to be $1127 \mathrm{~kg} \cdot \mathrm{m}^{-3}$. Solution viscosity and refractive index were estimated to be $6.0 \times 10^{-3}$ Pa.s and 1.3328 respectively $[11,12]$.

To estimate the electrophoretic mobility of tracer particles in the test solution, zeta potential was measured using DelsaNano HC Particle Analyser (Beckman Coulter, UK). Additionally, the zeta potential of $1 \mu \mathrm{m}$ borosilicate glass particles in the solution was also measured using Malvern Zetasizer Zs (Malvern Instruments, UK) to estimate the charge at the glass rod-liquid interface.

\subsection{Experimental device}

A rectangular film holder for electrokinetic flow investigations was fabricated by joining two clear borosilicate glass rods of diameter $3 \mathrm{~mm}$ with platinised titanium rods of diameter $2 \mathrm{~mm}$ (Ti-shop, William Gregor Ltd, UK) as shown in Figure 1(a). The rods were positioned to give internal dimensions of the flow cell to be $7.5 \mathrm{~mm} \times 1.4 \mathrm{~mm}$, where the liquid film is to be suspended.

Before all experiments, a film holder was thoroughly cleaned with Milli-Q deionised water. The frame was initially wetted with the test solution and the excess liquid adhered to the frame were 
removed using a short burst of compressed air. Following the frame preparation, a free liquid film was formed within the rods by dispensing $9 \mu \mathrm{L}$ of test solution using a micropipette (eppendorf) while gently moving the pipette tip along the cavity. The liquid film formed here is in direct contact with the parallel platinum electrodes positioned $7.5 \mathrm{~mm}$ apart and bounded by glass rods from the other two sides (Figure 1(b)). The top and the bottom interfaces of the film is open to the atmosphere (Figure 1(c)) while the free liquid film was suspended in the cavity by surface tension forces. Following the formation of the film, the holder was immediately transferred to the microscope stage (Figure 1(d)), and electrodes were connected to a DC power source (Thurlby Thandar PL30QMD). Flow is induced within the free liquid film when sufficient electric field was applied to the electrodes.

\subsection{Determination of film geometry}

Before electrokinetic experiments, the flow cell was scanned using CLSM (Nikon inverted Microscope ECLIPSE TE300 with Bio-Rad RAD200 scan head and 10X-0.25NA and 20X-0.45NA objective lenses) to determine the complex film geometry formed within the rods and to estimate the reproducibility of film dimensions. The boundaries of the liquid domain were clearly identified by the presence of Rhodamine B. For this purpose, 231 depth wise Image planes were recorded $4 \mu \mathrm{m}$ apart at various sections of the film to cover the whole domain. Finally, a 3D image of the flow cell was reconstructed by stacking all the images using ImageJ software as shown in Figure 1(b).

Electrokinetic flow experiments were carried out by establishing an external DC electric field between the platinum electrodes. For the preliminary experiments, the voltage was varied from $5 \mathrm{~V}$ to $20 \mathrm{~V}$. Following these initial range finding experiments, the applied voltage was kept constant at $12 \mathrm{~V}$ for the all experiments. Flow visualisation recordings were made in the mid-section of the film ( $5760 \mu \mathrm{m}$ away from the anode) at various depths to reduce the number of experimental 
dependencies presented. The recording commenced after $5 \mathrm{~s}$ following the application of an electric field. The system was relaxed for $30 \mathrm{~s}$ with no applied electric field before the next depthwise plane was recorded. All experiments were run at $20^{\circ} \mathrm{C}$.

\subsection{Flow visualisation}

The velocity field within the free liquid film due to the external electric field was measured using a CLSM $\mu$-PIV setup. The system consists of CLSM scan head, argon-ion laser (wavelength $=488 \mathrm{~nm}$, $100 \mathrm{~mJ}$ ), photomultiplier tubes ( 256 X 256 pixels, 8-bit resolution) and a computer with Laser Sharp 2000 software (see Figure 1). Fluorescent seeding particles $\left(\rho_{p}=1005 \mathrm{~kg} \mathrm{~m}^{-3}\right)$ of diameter $2 \mu \mathrm{m}$ with an excitation peak wavelength of $488 \mathrm{~nm}$ and emission peak wavelength of $530 \mathrm{~nm}$ were used for flow measurements. Following the identification of top and bottom interfaces of the liquid film using Rhodamine B, flow field was optically scanned at different depths $30 \mu \mathrm{m}$ apart. For each depth, 10 consecutive Images were recorded at a frame rate of $2 \mathrm{~Hz}$ with either 10X-0.25NA or 20X-0.45NA objective lenses with a field of view of $1350 \mu \mathrm{m} \times 1350 \mu \mathrm{m}$ for the former and $690 \mu \mathrm{m} \times 690 \mu \mathrm{m}$ for the latter. The optical slice thickness for this setup was estimated to be $8.4 \mu \mathrm{m}$ using Cytodex $^{\circledR} 3$ microcarrier beads (Sigma-Aldrich Ltd, UK) suspended in $30 \mathrm{mM}$ fluorescein isothiocyanate (FICT) solution. Recorded images were stored in the computer and analysed using software - PIVlab 1.4 and Matlab ${ }^{\mathrm{TM}}$ [13]. Cross-correlation analysis was performed by selecting 32 pixels $X 32$ pixels interrogation windows with $50 \%$ overlap to obtain a spatial resolution of $20 \mu \mathrm{m} \times 20 \mu \mathrm{m} \times 8.4 \mu \mathrm{m}$.

\subsection{Determination of the velocity field}

The measured velocity field from the initial $\mu$-PIV analysis consists of electroosmotic flow in the film, electrophoresis of tracer particles; pressure-driven flow and Brownian motion of the tracer particles. For the low Reynolds number flow under investigation, the resultant velocity was assumed to be a linear superposition of its components expressed as [14]:

This article is protected by copyright. All rights reserved. 


$$
\boldsymbol{u}_{m=} \boldsymbol{u}_{P}+\boldsymbol{u}_{E O F}\left(\zeta_{S L}, \zeta_{G L}\right)+\boldsymbol{u}_{E P}\left(\zeta_{p}\right)+\boldsymbol{d}_{b m} / \Delta t
$$

where $\boldsymbol{u}_{m}$ is the measured particle velocity, $\boldsymbol{u}_{P}$ is the velocity due to pressure, $\boldsymbol{u}_{E O F}$ is the velocity due to electroosmosis, $\boldsymbol{u}_{E P}$ is the particle electrophoretic velocity and $\boldsymbol{d}_{b m}$ is the displacement vector due to Brownian motion during the observation period $\Delta t$. The particle electrophoretic velocity, $\boldsymbol{u}_{E P}$, is a function of zeta potential at the seed particle surface $\left(\zeta_{p}\right)$ and the velocity due to electroosmosis, $\boldsymbol{u}_{E O F}$, is a function of zeta potential at the solid-liquid $\left(\zeta_{S L}\right)$ and gas-liquid interfaces $\left(\zeta_{G L}\right)$. For $2 \mu \mathrm{m}$ seeding particles, contribution due to Brownian motion can be neglected. Since the electrophoretic mobility of the particles can be measured separately, fluid velocity in the film can be calculated using the following equation.

$$
\boldsymbol{u}_{f}=\boldsymbol{u}_{m}-\boldsymbol{u}_{E P}\left(\zeta_{p}\right)
$$

where $\boldsymbol{u}_{f}$ is the combined fluid velocity due to electroosmotic flow and pressure flow expressed as

$$
\boldsymbol{u}_{f}=\boldsymbol{u}_{E O F}\left(\zeta_{S L}, \zeta_{G L}\right)+\boldsymbol{u}_{P}
$$

\subsection{Flow simulations}

The governing equations for fluid flow are continuity and incompressible Navier-Stokes equations for laminar flow (stokes flow);

$$
\begin{gathered}
\nabla \cdot\left[-p I+\mu(\nabla u)^{T}\right]=0 \\
\nabla \cdot u=0
\end{gathered}
$$

where $p$ is the pressure, $u$ is the divergence free velocity $((\nabla \cdot u)=0), \mu$ is the dynamic viscosity and $I$ is the identity matrix. The gas-liquid and solid-liquid interfaces were assumed to have the same slip velocity as both interfaces were covered by the surfactants. The slip velocity, $\boldsymbol{u}_{s}$, was determined according to Helmholtz-Smoluchowski relationship [15] given by;

$$
\boldsymbol{u}_{s}=\varepsilon \zeta \boldsymbol{E} / \mu
$$

where $\boldsymbol{E}$ is the electric field, $\varepsilon$ is the solution permittivity and $\zeta$ is the zeta potential at the surfactant covered interfaces.

The electric field inside the free liquid film was determined by following equations. 


$$
\begin{gathered}
\boldsymbol{E}=-\nabla \phi \\
\nabla \cdot\left(\varepsilon_{0} \varepsilon_{r} \boldsymbol{E}\right)=\rho_{v}
\end{gathered}
$$

where $\phi$ is the electric potential, $\varepsilon_{0}$ is the permittivity of vacuum, $\varepsilon_{r}$ is the relative permittivity of the liquid and $\rho_{v}$ is the space charge density. The boundary conditions are (i) electric potential at the liquid-electrode interface, i.e. $\phi=12 \vee$ and $\phi=0 \vee$ (ground electrode) (ii) zero electric charge at the glass-liquid and gas-liquid interfaces. For an interface covered with positively charged surfactant molecules, negatively charged ions accumulate in the vicinity of the interface to balance the charge; hence it allows assuming zero net charge at these interfaces. The deformation of the gas-liquid interfaces due to electric normal stresses were neglected compared to surface tension forces.

Numerical simulations were performed using a commercial finite element code Comsol Multiphysics $^{\top \mathrm{M}}$ 5.0. The 3D computational geometry generated from CLSM (Figure 1(c)) was imported to Comsol interface using CAD Import Module and discretised using tetrahedral mesh elements. Mesh-independency of the solution was confirmed using a higher mesh density of 811,927 elements. The number of degrees of freedom (DOFs) solved for was $5,564,874$. The computational time was approximately 5 hrs on an Intel Core i7 64-bit $2.7 \mathrm{GHz}$ processor.

\section{Results and Discussion}

Preliminary experiments were carried out with a range of voltages from $2 \mathrm{~V}$ to $35 \mathrm{~V}$ to identify suitable electric field strength for electrokinetic flow experiments. These voltages correspond to average electric field strengths of $254 \mathrm{~V} \cdot \mathrm{m}^{-1}$ to $4450 \mathrm{~V} \cdot \mathrm{m}^{-1}$ respectively. It was observed that high voltages caused significant electrolysis at the electrodes causing bubbles to form and disrupt the flow patterns while low voltages were insufficient to move the liquid in a consistent manner. Therefore, $12 \mathrm{~V}$ was selected for all electrokinetic flow experiments, which corresponds to an electric field of $1600 \mathrm{~V} \cdot \mathrm{m}^{-1}$. 
The ionic strength of the test solution was estimated to be $5 \mathrm{mM}$ and the corresponding Debye length was estimated to be $4 \mathrm{~nm}$ using Equation (9) [16].

$$
\lambda_{D}=\sqrt{\frac{\varepsilon R T}{2 F^{2} I_{C}}}
$$

where $R$ is the universal gas constant; $T$ is the temperature in Kelvin; $F$ is the Faraday constant and $I_{C}$ is the ionic strength of the solution. The ratio of Debye length to characteristic length scale of the flow is small enough to be neglected in this case [17] because the films investigated were relatively thick $(\sim 180 \mu \mathrm{m})$ compared to the thickness of the electrical double layer (EDL).

The following relationship gives the electrophoretic mobility for a spherical tracer particle with a uniform zeta potential [18]:

$$
\mu_{\mathrm{EP}}=\frac{2 \varepsilon \zeta_{p}}{3 \mu}
$$

The equation is valid for the case when the EDL is much thinner than the particle radius, which is the case under the experimental conditions used. Even though $\varepsilon$ and $\eta$ can vary within the flow domain, particularly in the vicinity of the interfaces, material properties were assumed to be constant throughout the film. The measured zeta potential of the fluorescent tracer particles used in the experiments for MTAB and SDS were $22 \pm 4 \mathrm{mV}$ and $-31 \pm 2 \mathrm{mV}$ respectively. The corresponding electrophoretic mobility determined using Equation (10) for MTAB and SDS were $1.40 \times 10^{-9} \mathrm{~m}^{2} \mathrm{~V}^{-1} \mathrm{~s}$ ${ }^{1}$ and $-1.97 \times 10^{-9} \mathrm{~m}^{2} \mathrm{~V}^{-1} \mathrm{~s}^{-1}$ respectively. The resulting electrophoretic drift velocity is given by:

$$
\mathbf{u}_{\mathrm{EP}}\left(\zeta_{p}\right)=\mu_{\mathrm{EP}} \mathbf{E}
$$

The fluid velocity due to electroosmotic flow and pressure build-up was obtained using Equations (2) by subtracting contribution from particle electrophoretic velocity calculated from equation (11) and shown in Figure 2(a). 
In computing the flow field using Finite Element Method (FEM), several assumptions were made.

First, the geometry of the flow cell determined by CLSM was assumed to be symmetric around half depth plane of the film. This was due to the opacity of the electrodes and thick glass rods restricting optical access to the top half of the film near the solid boundaries. However, due to gravitational forces liquid film tend to position towards the bottom of the rods making the geometry asymmetric. As a result of this shift in the film position, variations of the electric field can be expected in zdirection. Secondly, the net electric charges at the film interfaces were assumed to be zero for estimating the electric field as counter ions shield the charged surfactant heads. The Debye length at film interfaces were estimated to be on the order of few nanometers; therefore variation of charges at the boundaries is limited to a very narrow region. Then, the permittivity of the fluid was assumed to be uniform throughout the computational domain. Even though material properties change within the EDL [8], this can be neglected for a very thin EDL as estimated above.

Figure 2 compares the experimental and computational depthwise velocity profiles at the bottom half of the film for MTAB. Since the flow field at mid-film region is predominantly in the direction of the electric field, the plot shows fluid velocity in y direction (v) for half of the liquid film. The depthwise velocity profiles were extracted from confocal $\mu$-PIV measurements performed $5760 \mu \mathrm{m}$ away from the anode electrodes and the numerical solutions were extracted at the same locations for comparison. As the EDLs are formed at the cationic surfactant covered interfaces, electroosmotic flow dominated near the boundaries of the film towards the anode. However, the free liquid film is suspended in a frame and forms a closed-end flow cell. As a consequence, backpressure builds-up at the anode causing a flow towards the cathode in the core of the liquid film. This flow behaviour is observed for all the velocity profiles shown in Figure 2(a), but the flow magnitude shifted with depthwise location. 
Overall, the experimental data measured by $\mu$-PIV matched well with the velocity profiles predicted by the numerical simulations. The velocity magnitude and the flow features are reasonably close, but a clear shift was observed towards the top half of the liquid film. A possible cause for this shift is the assumptions made during geometry construction of the model. Due to the difficulty in optically slicing the film near the solid rods, it was assumed that the centre of the film was located in the centre of the frame rods making the geometry perfectly symmetrical around the mid-depth plane. This assumption leads to a uniform electric field at the mid region of the film far from electrodes. However, it was observed that the free liquid film tends to position towards the bottom half of the rod making the film geometry asymmetric around the mid-depth plane. As a consequence, the electric field will be stronger towards the top half of the film and electroosmotic flow will dominate in that region reducing any backflow. Therefore, backflow will be stronger towards the bottom of the film where film is wider than the top surface (see Figure 3 ).

In order to investigate the effect of surfactant type on the flow field, velocity profiles were compared for both cationic and anionic surfactants (Figure 4). The information presented in Figure 2 was for half of the film width and any asymmetry around the centre of the film were not captured. Therefore, this study was aimed at observing information for the entire width of the free liquid film as shown in Figure 4(a). For this purpose, a lower magnification of 10X was used instead of a 20X lens. This allowed the view of overall flow profile at the expense of losing resolution. Therefore, only the data from two surfactant types are presented at the mid-depth plane instead of a depthwise study. 
The dependencies shown in Figure 4 presents y component of the velocity $(v)$ non-dimensionalized by the maximum velocity at the centre of the film $\left(v_{\max }\right)$ for cationic surfactant MTAB and anionic surfactant SDS. Two main observations were noted. Firstly, the fluid flow profile observed in the free liquid films agreed well with that of a closed-end solid microchannels reported in literature [19] for both surfactants. However, for a solid-walled microchannel, charge distribution near the channel walls are determined by the wall material whereas for a film stabilised by surfactants, charge of the EDL depends on the charge of the surfactant head adsorbed. Secondly, when the type of surfactant was changed from cationic to anionic, the flow profile reversed the direction, but flow features remained the same. In the case when a cationic surfactant was used to make films, EDLs are predominantly formed of negative ions which resulted in the electroosmotic pumping of fluid towards anode electrode. However, when the cationic surfactant was replaced by an anionic surfactant such as SDS, EDLs are predominantly formed of positive ions, resulting electroosmotic pumping towards the cathode electrode. Hence, a possible arrangement of surfactants on the gasliquid and solid-liquid interfaces for a cationic surfactant (MTAB) and formation of corresponding EDL is shown in Figure 5.

Computational velocity profiles for cationic and anionic surfactants presented in Figure 4(b) closely match with the experimental results shown in Figure 4(a). This supports our proposition of possible arrangement of surfactants at the interfaces shown in Figure 5. For all the experiments, the surfactant concentration in test solutions was set to just above the $\mathrm{CMC}$ to have sufficient surfactant molecules to cover the film interfaces, but not to form a large number of micelles. Therefore, surfactant covered interfaces were assumed to be immobile in the tangential direction for numerical simulations. However, the validity of this assumption should be verified in future work to neglect viscoelastic flow at the interfaces. 


\subsection{Concluding Remarks}

Simulated velocity fields obtained using finite element method to model a free liquid film stabilised by anionic and cationic surfactants are compared with experimentally measured velocity profiles using confocal $\mu$-PIV. Experimental velocity profiles agree closely with computational predictions, but deviations were noted at the top half of the liquid film. These deviations were attributed to uncertainties associated with experimental measurements and assumptions applied to the computational model.

The asymmetry arising from the location of the free liquid film towards the bottom of the frame seems to have a greater effect than expected and should be considered constructing the computational domain. Overall, the velocity profiles for a free liquid film were similar to that of a closed-end microchannel, but the flow direction and flow features will depend on the type of surfactant used. Wet liquid foam under an applied electric field is a complex system to study, but understanding the nature of fluid flow in parts of such a system is important in exploiting foam electrokinetics for separating biological molecules such as proteins and DNA. The free liquid film investigated in this paper is similar to a foam Plateau border, however further work is required to fully understand the dynamic behaviour.

\section{Acknowledgements}

Authors would like to thank the Department of Chemical Engineering at Loughborough University for a doctoral scholarship for AHS; Marie Curie CoWet project, EU and MAP EVAPORATION, European Space Agency and Santander Mobility Award Scheme. The authors would also like to thank Mr. Tony Eyre for technical assistance and Dr. Shaghayegh Ghanizadeh for laboratory support. 


\section{References}

[1] Braide-Azikiwe, D.C.B., Holt, K.B., Williams, D.E., Caruana, D.J., Electrochem. Commun. 2009, $11,1226-1229$.

[2] Nasiri, M., Shirsavar, R., Saghaei, T., Ramos, A., Microfluid. Nanofluidics. 2015, 19, 133-139.

[3] Feiz, M.S., Namin, R.M., Amjadi, A., Phys. Rev. E, 2015, 92, 0330021-9.

[4] Shirsavar, R., Ramos, A., Amjadi, A., Taherinia, J., Mashhadi, M., Nejati, A., J. Electrostat. $2015,73,112-116$.

[5] Bonhomme, O., Liot, O, Biance, A.-L., Bocquet, L., Phys. Rev. Lett. 2013, 110, 0545021-5.

[6] Bonhomme, O., Mounier, A., Simon, G, Biance, A.-L.A., J. Phys. Condens. Matter. 2015, 27, $1941181-7$.

[7] Sett, S., Sahu, R.P., Sinha-Ray, S., Yarin, A.L., Electrochim. Acta. 2016, 187, 693-703.

[8] Baldessari, F., Santiago, J.G., J. Nanobiotechnology. 2006, 4,12.

[9] Joly, L., Detcheverry, F., Biance, A.-L., Phys. Rev. Lett. 2014, 113, 0883011-5.

[10] Vrij, A., J. Colloid Sci. 1964, 19, 1-27.

[11] Segur, J.B., Oberstar, H.E., Ind. Eng. Chem. 1951, 43, 2117-2120.

[12] Leron, R.B., Soriano, A.N., Li, M.H., J. Taiwan Inst. Chem. Eng. 2012, 43, 551-557.

[13] Thielicke, W., Stamhuis, E.J., J. Open Res. Softw. 2014, 2:e30, 1-10.

[14] Devasenathipathy, S., Santiago, J.G., Takehara, K., Anal. Chem. 74, 3704-13.

[15] Hunter, R.J., Foundations of Colloid Science, 2nd ed., Oxford University Press, New York, 2001.

[16] Kirby, B.J., Micro- and Nanoscale Fluid Mechanics, CAMBRIDGE UNIVERSITY PRESS, Cambridge, 2010.

[17] Karniadakis, G., Beskok, A., Aluru, N., Microflows and Nanoflows Fundamentals and Simulation, Springer Science + Business Media, Inc., New York, 2005.

[18] Hunter, R.J., Zeta Potential in Colloid Science: Principles and Applications, Elsevier Science, 
1981.

[19] Ooi, K.T., Yang, C., Chai, J.C., Wong, T.N., 2005 43, 1349-1362.

Figure 1: Experimental setup (a) Free liquid frame holder; (b) Geometry of the freely suspended liquid film constructed by confocal microscopy scanning; (c) A cross section of the free liquid film depicting interfaces; (d) Confocal $\mu$-PIV setup for flow measurements.

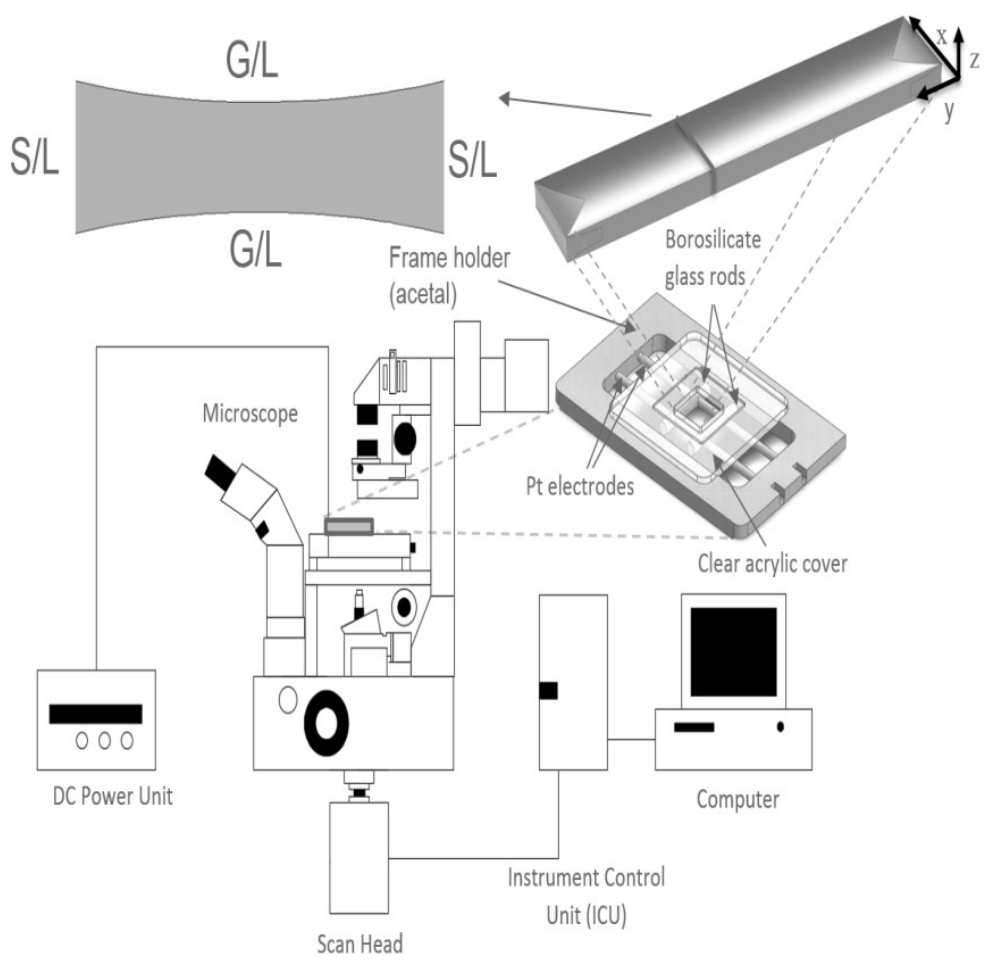

Figure 2: Depthwise velocity profiles due to combined pressure and electroosmotic flow in a free suspended liquid film stabilised using MTAB at a location $5760 \mu$ m away from the anode. (a) y component of the velocity measured by $\mu$-PIV (b) y-velocity computed from numerical simulations. Both data sets show half of the free liquid film width. $\mathrm{x}$ is measured from the centre of the film and the legend shows the micron depths at which the measurements were taken. 

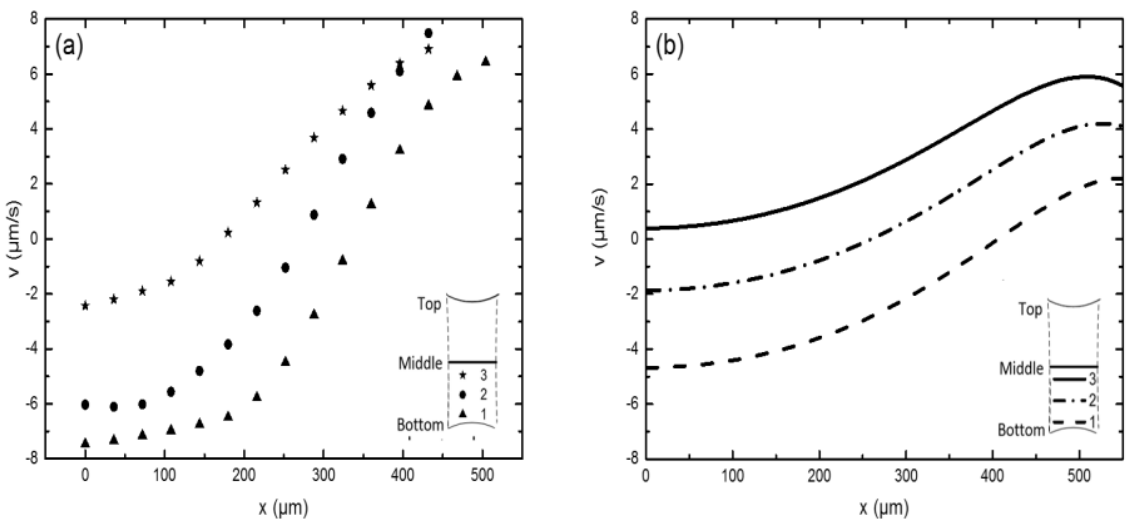

Figure 3: Combined electroosmotic and pressure flow profiles for a closed-end free liquid film with asymmetric geometry.

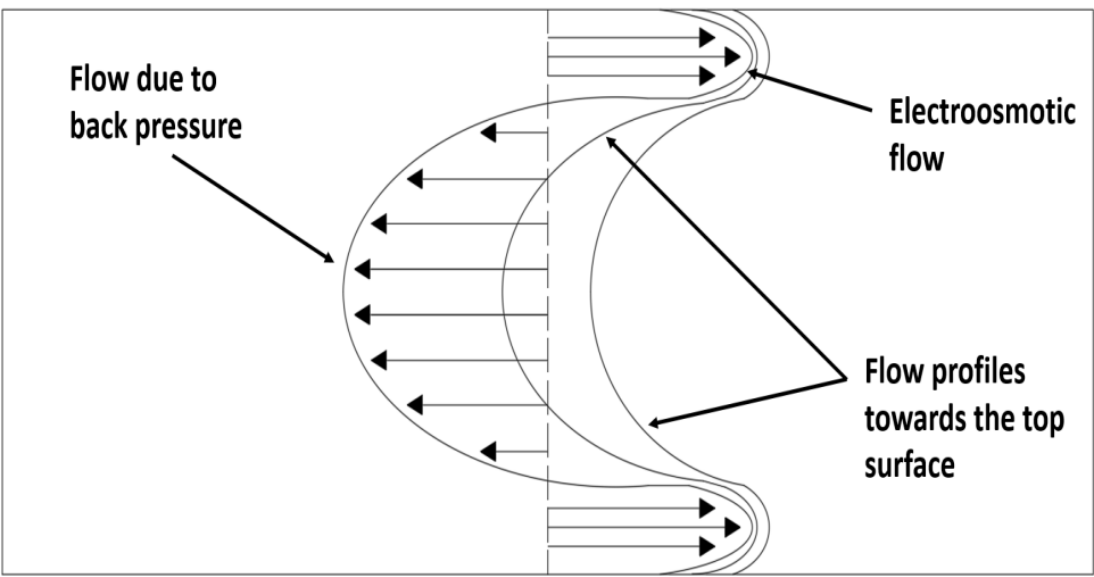

Figure 4: Comparison of flow profiles for free liquid films stabilised with cationic and anionic surfactants (a) Experimental measurements using $\mu$-PIV and (b) Numerical simulations. y velocity ( $v$ ) 
and lateral distances $(x)$ are non-dimentionalised using maximum y velocity at the centre of the film and half film width $(W)$ respectively.
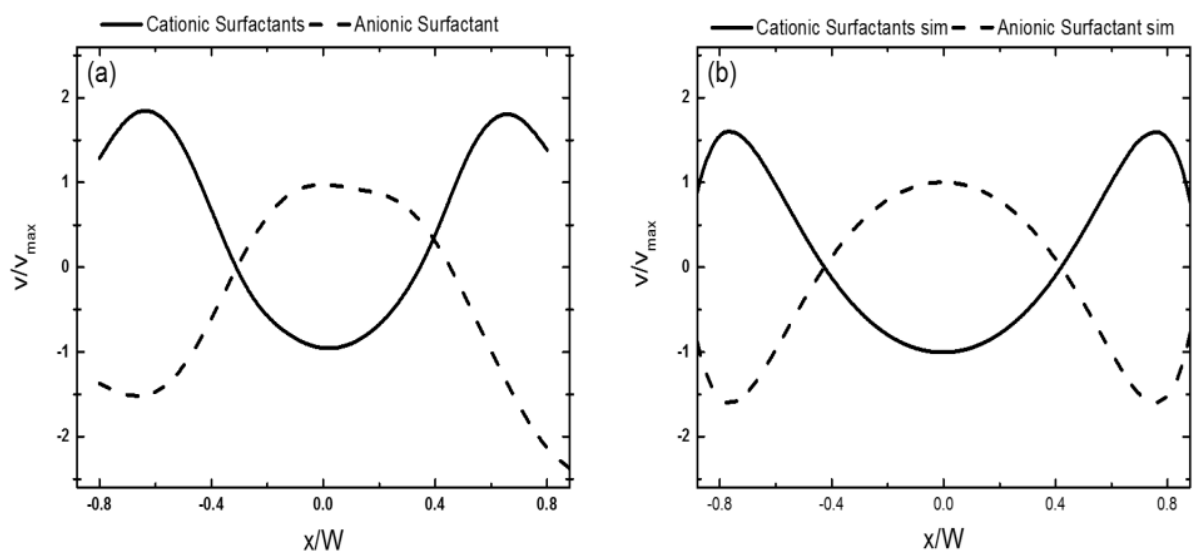

Figure 5: Schematic representation of the film cross-section depicting surfactant molecule arrangement (left) and illustration of the EDL at the interfaces (right). 

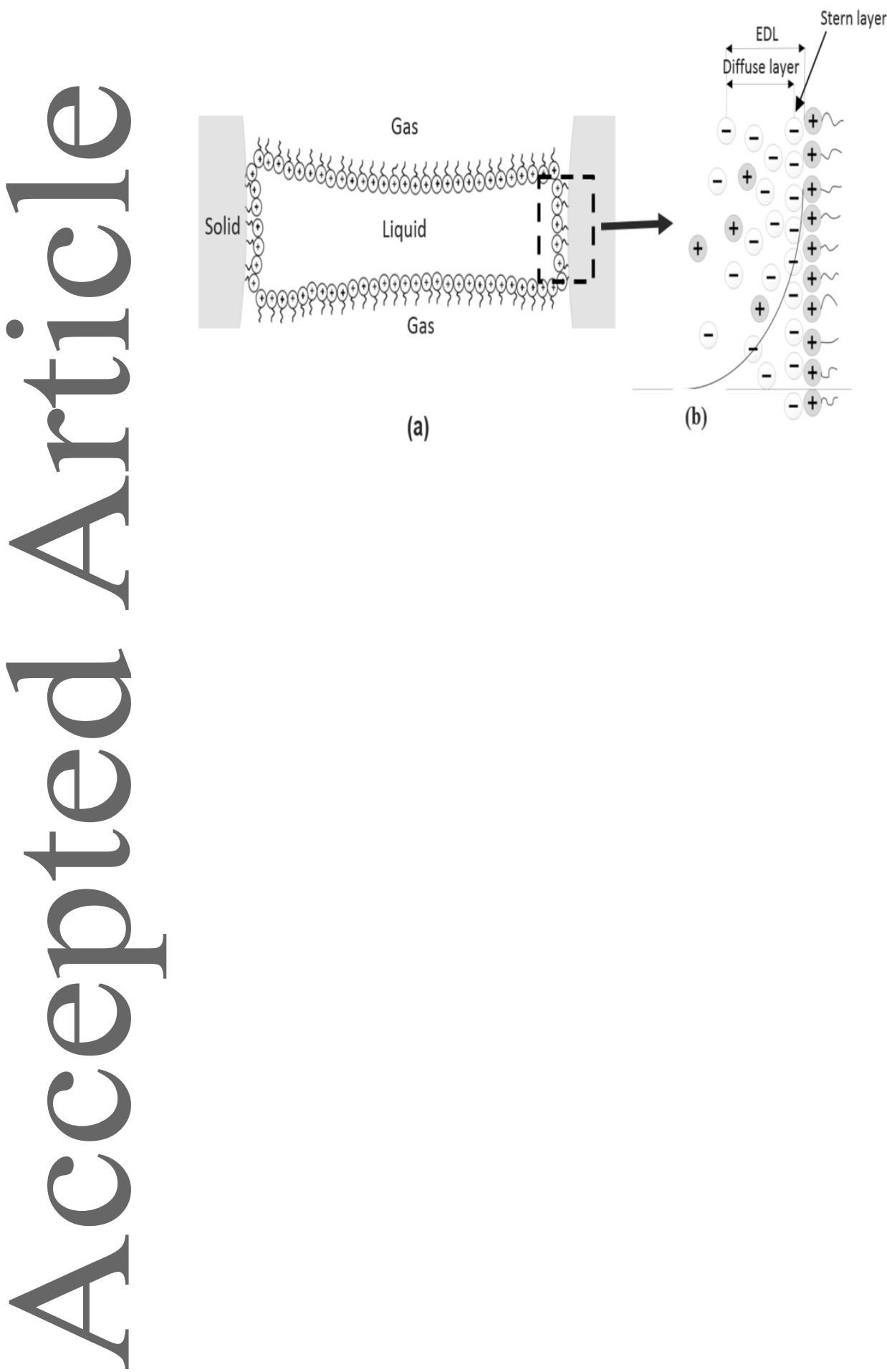

(a)

(b) 